\title{
Wavelength Tunable Erbium-Doped Fiber Ring Laser Based on Fiber Bragg Grating Xiaobo Zhou ${ }^{1, \text { a }}$ \\ ${ }^{1}$ Xijing University, Shaanxi 710123, China \\ a369146317@qq.com
}

\begin{abstract}
Designed a ring erbium-doped fiber laser based on the principle of wavelength tuning of FBG. Utilizing Fiber Bragg Grating(FBG) which encapsulated on PZT as a wavelength tunable component. The output lasing wavelength between $1550.24 \mathrm{~nm}$ and $1552.36 \mathrm{~nm}$ can be tuned continuously during $0 \sim 400 \mathrm{~V}$ voltage range. The tuning linearity is up to $99.97 \%$, the $3 \mathrm{~dB}$ linewidth is less than $0.04 \mathrm{~nm}$, the side mode suppression ratio(SMSR) is more than $63 \mathrm{~dB}$ and the output power of EDFL is up to $15.2 \mathrm{~mW}$.
\end{abstract}

Keywords: Fiber bragg grating(FBG);Erbium-doped fiber laser(EDFL);PZT

\section{基于光纤 Bragg 光栅调谐的掺铒光纤激光器}

\author{
周晓波 $1^{1}$
}

(1. 西京学院 机电技术系, 陕西 西安 710123)

摘要: 在介绍光纤光棚波长调谐原理的基础上设计了一种环形腔掺铒光纤激光器。利用封装在压电陶瓷 (PZT) 上的光 纤光棶 ( FBG) 作为波长调谐元件, 在 $0 \sim 400 \mathrm{~V}$ 的电压范围内, 实现了输出激光波长在 1550.24 1552.36nm 内的连续可调, 调谐线性度高达 $99.97 \%$ ，激光光谱的 $3 \mathrm{~dB}$ 带宽均小于 $0.04 \mathrm{~nm}$, 边模抑制比 (SMSR) 大于 $63 \mathrm{~dB}$, 输出功率可达 $15.2 \mathrm{~mW}$ 。

关键词：光纤光栅；掺铒光纤激光器（EDFL）；压电陶瓷（PZT）

中图分类号：TN 253 文献标志码：A

引言

目前通信用激光器主要是半导体激光器, 但半导体激光器存在着成本高, 与系统的传输光纤耦合困难 等缺点, 限制了它在通信系统中的应用, 而可调谐光纤激光器的应用除了可大大降低间隔为 $25 \mathrm{GHz}$ 的密集 波分复用（Dense Wavelength Division Multi Plexing）系统的运营成本和备份成本、实现用一个可调 谐激光器代替多个固定波长激光器作为备用外, 还有低阈值、泵浦效率高、激光波长位于 $1550 \mathrm{~nm}$ 通信窗 口和与光纤通信系统完全兼容等特点 ${ }^{[1-3]}$ 。

可调谐光纤激光器在光纤光栅传感作为传感扫描光源, 可实现单点、多点及分布式光纤光栅阵列的传 感检测, 并且随着窄带滤波技术的发展, 基于可调谐光纤激光器的波长检测技术将有望成为最具有发展潜 力的光纤光栅传感检测技术 ${ }^{[4]}$ 。

\section{1 光纤光栅传感原理}

根据光纤布拉格光栅的耦合模理论, 均匀非闪耀光纤布拉格光栅可将其中传输的一个导模耦合成沿另 一个相反方向传输的导模而形成窄带反射, 峰值反射布拉格波长 $\lambda_{B}$ 为:

$$
\lambda_{B}=n_{\text {eff }} \Lambda
$$


式中 $n_{\text {eff }}$ 为导模的有效折射率, $\Lambda$ 为光栅周期。

引起 $\lambda_{B}$ 漂移的因素有多种, 若只考虑应变 $\varepsilon_{\text {的影响, 则 } \lambda_{B} 、 n_{\text {eff }} 、 \Lambda \text { 只是 }} \varepsilon$ 的函数。当光纤 Bragg 光栅收到外力作用时, 将引起光栅周期的变化和弹光效应, Bragg 光栅反射波中心波长 $\lambda_{B}$ 将发生偏移, 即

$$
\Delta \lambda_{B}=2 \Delta n_{e f f} \Lambda+2 n_{e f f} \Delta \Lambda
$$

在轴向应力作用下, Bragg 光栅反射波中心波长相对偏移量与轴向应变之间

$$
\Delta \lambda_{B} / \lambda_{B}=\left(1-p_{e}\right) \varepsilon
$$

式中, $p_{e}$ 为光纤的有效光系数, $\varepsilon$ 为光纤 Bragg 光栅的轴向应变, 对于石英光纤 $p_{e}=0.216$, 应变灵 敏度系数为 0.748 。

再根据轴向应力与应变的关系可得中心波长相对偏移量与应力之间得关系为

$$
\Delta \lambda_{B} / \lambda_{B}=\frac{\left(1-p_{e}\right)}{E} \sigma
$$

式中, $\mathrm{E}$ 为光纤 Bragg 光栅得弹性模量, $\sigma$ 为轴向应力, $\left(1-p_{e}\right) / E$ 为应力灵敏度系数, 对于熔石英 光纤, $E=7.0 \times 10^{10} \mathrm{~Pa}$, 则其应力灵敏度系数为 $1.12 \times 10^{-11} \mathrm{~Pa}^{-1}$ 。

\section{PZT 调谐原理}

压电陶瓷（PZT）是一种具有压电性能的多晶体，是信息功能陶瓷的重要组成部分。压电陶瓷最大的 特性是具有正压电性和逆压电性。正压电性是指某些电介质在机械外力作用下, 介质内部正负电荷中心发 生相对位移而引起极化, 从而导致电介质两端表面内出现符号相反的束缚电荷。其电荷密度与外力成正比, 遵循公式:

$$
\delta=d T
$$

其中 $\delta$ 为面电荷密度, $\mathrm{d}$ 为压电常数, $\mathrm{T}$ 为伸缩应力。

反之, 当给具有压电性的电介质加上外电场时, 电介质内部正负电荷中心不但发生相对位移而被极化, 同时, 由于此位移而导致电介质发生形变, 这种效应称之为逆压电性。形变与外电场呈线性关系, 遵循公 式:

$$
\vec{x}=d_{t} \vec{E}_{s}
$$

其中 $d_{t}$ 为压电应变常数, $\vec{E}_{s}$ 为外加电场, $\vec{x}$ 为应变。

压电陶瓷是一种弹性体, 它服从胡克定律: “在弹性限度范围内, 应力与应变成正比”。设应力为 $\mathrm{T}$, 加于截面积 $\mathrm{A}$ 的压电陶瓷片上, 其所产生的应变为 $\mathrm{S}$, 则根据胡克定律, 应力 $\mathrm{T}$ 与应变 $\mathrm{S}$ 之间有如下关系 $\mathrm{S}=\mathrm{sT}, \mathrm{T}=\mathrm{cS}$, 式中, $\mathrm{S}$ 为弹性顺度常数, 单位为 $\mathrm{m} 2 / \mathrm{N} ; \mathrm{C}$ 为弹性劲度常数, 单位为 $\mathrm{N} / \mathrm{m} 2$ 。

\section{3 结构及工作原理}

可调谐掺铒光纤激光器是基于掺铒光纤荧光放大原理, 在光纤激光器回路中加入可调谐滤波器形成波 长反馈, 控制激光器的激射波长实现波长调谐的。所谓的光纤 Bragg 光栅的波长调谐是指对制作好的 FBG 
通过不同的物理效应（热膨胀效应、热光效应及弹光效应）改变 FBG 光栅周期及光栅折射率分布，使反射 波长产生一定量的漂移, 以达到调谐 FBG 反射（透射）波长的目的。

实验装置如图 1 所示, 构成以反射波长可调光纤光栅作为端面反射镜的环形结构的掺铒光纤激光器。 环形腔由波分复用器 (WDM)、掺铒光纤 (EDF)、隔离器 (ISO)、宽带耦合器 $(B B C)$ 、光环行器 $(O C)$ 等组 成。980nm 抽运光通过 $980 \mathrm{~nm} / 1550 \mathrm{~nm}$ 波分复用器有效的耦合到掺铒光纤中。实验所用掺铒光纤模场直径为 $0.20 \mu \mathrm{m}$, 截止波长为 $960 \mathrm{~nm}, 980 \mathrm{~nm}$ 峰值吸收为 $7.6 \mathrm{~dB} / \mathrm{m}, 1530 \mathrm{~nm}$ 的近峰值吸收为 $15.2 \mathrm{~dB} / \mathrm{m}$, 经过优化 所选用的光纤长度为 $12 \mathrm{~m}$ 。光沿着环行器 1 至 2,2 至 3 的方向单向传播。在 2 端口, 我们接入一根中心 波长为 $1550.12 \mathrm{~nm}$ 的光纤光栅, 加上 $1 \mathrm{~N}$ 的预应力（波长漂移至 $1550.24 \mathrm{~nm}$ ), 并将其粘贴在压电陶瓷位移 器的表面。实验中所用的压电陶瓷位移器为压电陶瓷构成层叠式高精度位移器, 型号为 PT200, 尺寸为 $8 \times 8 \times 60 \mathrm{~mm}$, 最大标称位移为 $60 \mu \mathrm{m}$, 最大推力 $900 \mathrm{~N}$, 刚度 $30 \mathrm{~N} / \mu \mathrm{m}$ 。

当给压电陶瓷加上驱动电压时, 由于逆压电效应，压电陶瓷将随之产生形变，进而施加应变在光纤光 栅上，使得光纤光栅的反射波长发生漂移，实现对光纤光栅的调谐。通过对 PZT 所加电压的调节来实现 FBG 反馈波长的改变, 可实现不同波长的光的激射。环形腔中光隔离器的作用是使光单向循环, 避免双向激射, 发生模式竞争，折射率匹配液（IMG）的作用是防止光纤的端面反射。

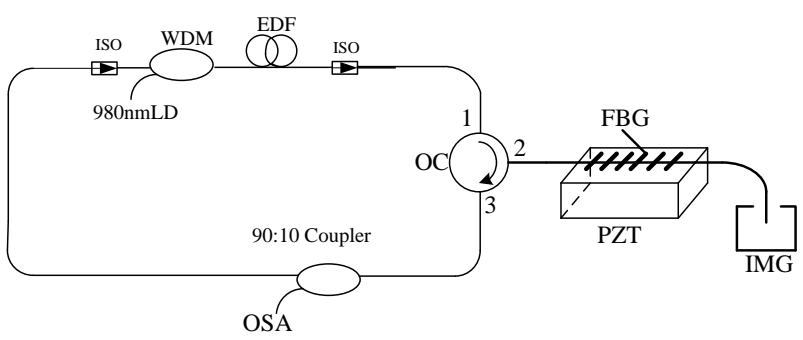

图 1 基于 PZT 调谐的掺铒光纤激光器结构

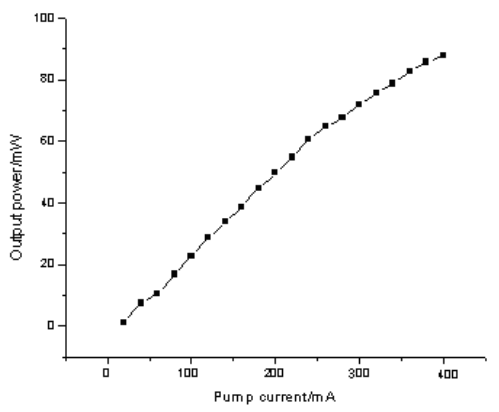

图 2 LD 输出功率与洜浦电流的关系

此结构的光纤激光器的基本原理是：掺有 $E r^{3+}$ 的光纤在 $980 \mathrm{~nm}$ 泵浦光的作用下, 使 $E r$ 粒子从低能级 跃迁到高能级, 实现粒子数反转分布, 在没有激励信号光输入的情况下, 部分粒子将衰变回基态, 形成自 发辐射。自发辐射产生的苂光通过 FBG 反射形成窄带光并被反馈进入掺铒光纤充当信号光。在 $980 \mathrm{~nm}$ 激光 二极管的抽运下, 铒粒子发生粒子数反转且受激发射大于受激吸收时, 信号光将被放大, 再经过掺铒光纤 中的往复传播形成激光。通过电压变化调谐光纤光栅的反射波长达到改变反馈信号光的波长, 可形成不同 波长的单纵模激光输出, 实现该掺铒光纤激光器的波长可调谐输出。

\section{4 实验结果及分析}

实验中，抽运光源采用中心波长为 $979.04 \mathrm{~nm}$ 的 $980 \mathrm{~nm}$ LD，阈值电流为 $30 \mathrm{~mA}, \mathrm{LD}$ 尾纤输出光功率随抽 运电流的变化关系如图 2 所示, 当驱动电流大于 $30 \mathrm{~mA}$ 时, 开始有功率输出, 而随着驱动电流的增大, $\mathrm{LD}$ 输出功率基本呈线性增加, 当达到 $300 \mathrm{~mA}$ 后逐渐趋于饱和。考虑到输出耦合比对激光输出性能的影响 ${ }^{[5]}$, 我们最终选用 90: 10 的光耦合器, 并在 10\%端口进行光谱测量, 激光器的输出功率随着泵浦功率的增加 基本成线性关系。

在保持激光器结构不变、驱动电流固定在 $200 \mathrm{~mA}$ （输出泵浦功率为 $85 \mathrm{Mw}$ ）情况下，使用粘贴在层叠式 
压电陶瓷位移器表面的光纤光栅作为激光器的端面反射镜以实现波长反馈。通过对压电陶瓷施加不同的直 流电压来实现光纤激光器的波长调谐, 利用多功能光谱仪对激光器的输出光谱进行监测, 光谱仪最小分辨 率为 $0.05 \mathrm{~nm}$ 。实验中, 对压电陶瓷施加 $0 \sim 400 \mathrm{~V}$ 的直流电压, 在未加电压时测得激光输出谱线如图 3a 所 示, 中心波长为 $1550.24 \mathrm{~nm}$, 输出功率可达 $15.2 \mathrm{~mW}$, 边模抑制比为 $63 \mathrm{~dB}, 3 \mathrm{~dB}$ 带宽小于 $0.04 \mathrm{~nm}$ 。当电压加 到最大 $400 \mathrm{~V}$ 时, 激光器的输出光谱如图 $3 \mathrm{~b}$ 所示, 此时由于压电陶瓷的逆压电效应, 使得粘贴在压电陶瓷 位移器上的光纤光栅产生轴向应变, 中心波长发生漂移, 反馈进激光器环形腔后使得输出激光的波长发生 改变。激光输出中心波长为 $1552.36 \mathrm{~nm}$, 激光谱形与未加电压相比, 基本上保持不变。

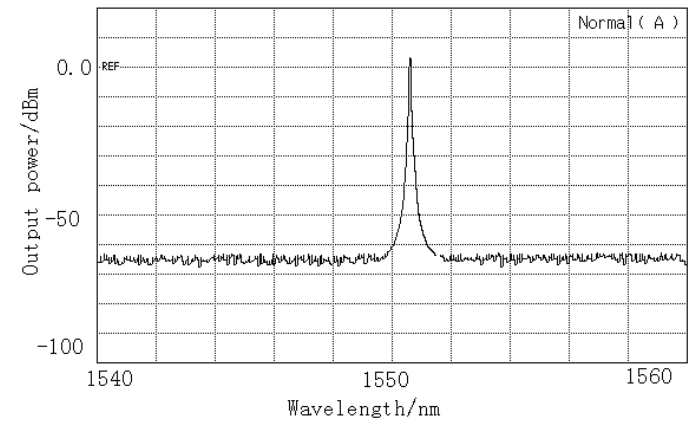

(a) 未加电压时激光输出谱线

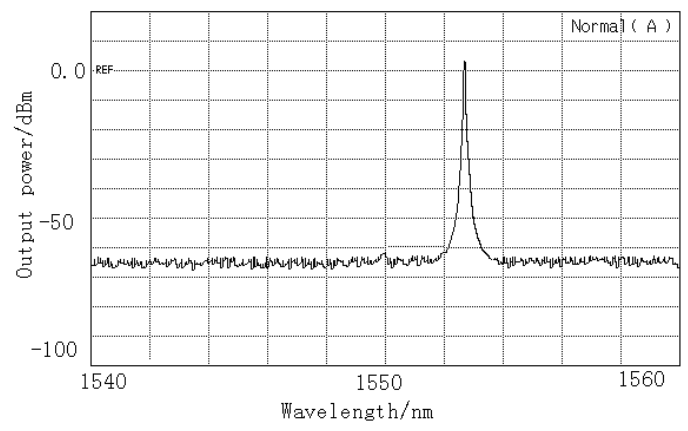

(b) $400 \mathrm{~V}$ 电压时激光输出谱线

图 3 不同电压激光器输出

通过连续调谐 PZT 上电压, 同时利用光谱仪对其进行监测, 测得激光器输出光谱的峰值波长与电压变 化曲线如图 4 所示。在 $0 \sim 400 \mathrm{~V}$ 的电压变化范围内, 波长调谐范围为 $2.12 \mathrm{~nm}$, 线性度为 $99.97 \%$ 。同时观 察到: 在对光栅的调谐过程中, 不同波长的输出激光形状基本保持图 5 (200V 时的输出激光谱) 所示谱型, 没有发现多波长激射现象, 激光光谱的 $3 \mathrm{~dB}$ 带宽小于 $0.04 \mathrm{~nm}, 20 \mathrm{~dB}$ 带宽均小于 $0.1 \mathrm{~nm}$, 边模抑制比高达 $63 \mathrm{~dB}$, 功率稳定性优于 $0.1 \mathrm{dBm}$ 。经过多次重复测量, 误差在仪器测量误差范围内, 而且具有较高的线性度、 较好的重复性, 还具有保持功率输出的平坦等特点, 在众多的调谐方法中, 可以说是一种简单且有效的调 谐方法。

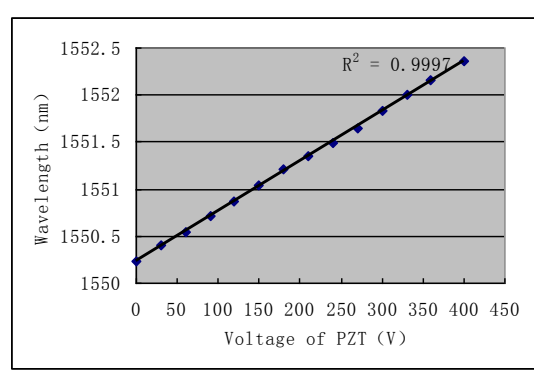

图 4 光纤激光器电压调谐曲线

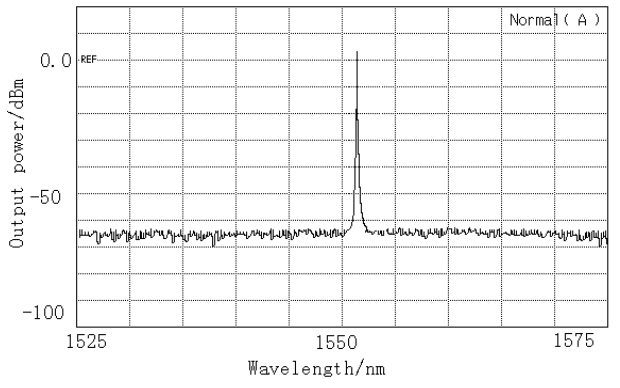

图 5 电压为 $200 \mathrm{~V}$ 的激光输出光谱

通过实验看出, PZT 电压调谐范围要比其它调谐方式 (温度、压力、悬臂梁等调谐) 的调谐范围小的 多, 但是它具有调谐方式简单、调谐方便、线性度好、易于集成等优点。若将实验中的光纤光栅换用具有 不同中心波长的光纤光栅作为端面反射镜, 便可实现输出波长在 $1525 \sim 1565 \mathrm{~nm}$ 范围内的输出, 覆盖通信 的 $\mathrm{C}$ 波段范围。因此, 在光纤通信系统中若利用此种波长可调谐光纤激光器充当光源, 就可以避免每一个 波长要用一个激光器的现象, 这样可以大大减少固定波长激光器的数量, 在和大程度上降低系统成本, 这 就要求激光器的线宽和稳定性达到固定激光器的要求, 而对于调谐速率, 我们发现通过电压调谐, 其速率 远远大于其它调谐方式, 完全能满足系统要求。因此下一步工作的重点是实现激光器性能的高稳定。 


\section{5 结论(结语)}

设计了一种结构简单的环形掺铒光纤激光器, 在激光器环形腔内采用光纤光栅反馈滤波实现了连续调 谐窄带激光输出, 通过改变 PZT 电压实现对激光器进行调谐。实现了 $1550.24 \sim 1552.36 \mathrm{~nm}$ 波长范围内的 窄线宽高功率波长可调谐激光输出, 调谐线性度高达 $99.97 \%$, 激光 $3 \mathrm{~dB}$ 带宽小于 $0.04 \mathrm{~nm}$, 边模抑制比可 达 $63 \mathrm{~dB}$, 功率稳定性由于 $0.1 \mathrm{dBm}$, 充分证实了基于光纤光栅的可调谐波长输出的光纤激光器的技术方法 的可行性及其优势。以此, 这种光纤激光器必将在光纤通信及光纤传感领域得到广泛应用。

\section{6 致谢}

陕西省教育厅专项基金 (2013JK1121); 西京学院科研基金(XJ120235); 西京学院科研基金 (XJ140236)。

\section{Acknowledgement}

Shaanxi Department of Education Special Research Project(2013JK1121);Xijing University Scientific Research Foundation Project(XJ120235); Xijing University Scientific Research Foundation Project(XJ140236).

\section{参考文献:}

[1] MEL0 M, FRAZA0 0, REIXEIRA A L J, et al. Tunable L-band erbium-doped fiber ring laser by means of induced cavity loss using a fiber taper[J]. Appl. Phys , 2003, 30(7): 139-142.

[2] 张建生, 郭玉涁, 王天枢. 可编程控制波长调谐的环形掺铒光纤激光器 [J]. 半导体光电, 2004, 25 (5): 366-369.

[3] 习聪玲. 多波长超宽带铒铥混合掺杂光纤光源 [J]. 光通信技术, 2014, (02) : 43-45.

[4] 刘颖刚, 乔学光, 贾振安, 等. 一种高性能光纤 ASE 光源的优化与研究 [J]. 光电子. 激光, 2011, (10) : 1475-1478.

[5] 刘颖刚, 车伏龙, 傅海威, 等. 光纤布拉格光棚折射率传感研究进展 [J]. 光电技术应用, 2015, (01):1-7.

[6] 李建鹏, 曾晓琳, 官静雯, 等. 光纤布拉格光栅的折射率传感研究 [J].激光与红外, 2016, (06) :737-741.

\section{References}

[1] MELO M, FRAZAO O, REIXEIRA A L J, et al. Tunable L-band erbium-doped fiber ring laser by means of induced cavity loss using a fiber taper[J]. Appl. Phys, 2003, 30(7): 139-142.

[2] Zhang Jing-sheng, Guo Yu-bin, Wang Tian-shu. Progammable Wavelength Tunable Erbium-doped Fiber Ring Laser[J].Semiconductor Optoelectronics, Vol.25(2004), No.5:366-369 (in Chinese)

[3] Xi Cong-ling. Study of an ultra-wideband multi-band hybrid-doped fiber light source [J]. Study on Optical Communications, Vol. 2(2014), 43-45 (in Chinese)

[4] Liu Ying-gang, Qiao Xue-guang, Jia Zhen-an, et al. Optimization and Study on a High-performance ASE Fiber Source [J].Journal of Optoelectronics. Laser, Vol.22(2011), No.10, 1475-1478 (in Chinese)

[5] Liu Ying-gang, Che Fu-long, Fu Hai-wei, et al. Research Progress of Fiber Bragg Grating Refractive Index Sensing[J]. Electro-optic Technology Application, Vol.30(2015),No.1, 1-7 (in Chinese)

[6] Li Jian-peng, Zeng Xiao-lin, Guan Jing-wen, et al. Research on Refractive Index of Fiber Bragg Grating Sensor[J].Laser \&Infrared, Vol.46(2016),No.6, 737-741 (in Chinese) 